\title{
Solution Conditions Tune and Optimize Loading of Therapeutic Polyelectrolytes into Layer-by-Layer Functionalized Liposomes
}

\author{
Santiago Correa ${ }^{\dagger, \#, \perp}$, Natalie Boehnke ${ }^{\ddagger, \#}$, Elad Deiss-Yehiely ${ }^{\S}$ Paula T. Hammond ${ }^{\star}$,,$\|$ \\ tDepartment of Biological Engineering, Massachusetts Institute of Technology, 21 Ames Street, \\ Cambridge, Massachusetts 02142, United States \\ ¥Koch Institute for Integrative Cancer Research, Massachusetts Institute of Technology, 500 Main \\ Street, Cambridge, Massachusetts 02142, United States \\ §Department of Materials Science and Engineering, Massachusetts Institute of Technology, 183 \\ Memorial Drive, Cambridge, Massachusetts 02142, United States \\ "Department of Chemical Engineering, Massachusetts Institute of Technology, 25 Ames Street, \\ Cambridge, Massachusetts 02142, United States
}

\begin{abstract}
Layer-by-layer ( $\mathrm{LbL}$ ) nanoparticles offer great potential to the field of drug delivery, where these nanocomposites have been studied for their ability to deliver chemotherapeutic agents, small molecule inhibitors, and nucleic acids. Most exciting is their ability to encapsulate multiple functional elements, which allow nanocarriers to deliver complex combination therapies with staged release. However, relative to planar LbL constructs, colloidal LbL systems have not undergone extensive systematic studies that outline critical synthetic solution conditions needed for robust and efficient assembly. The multistaged process of adsorbing a series of materials onto a nanoscopic template is inherently complex, and facilitating the self-assembly of these materials depends on identifying proper solution conditions for each synthetic step and adsorbed material. Here, we focus on addressing some of the fundamental questions that must be answered in order to obtain a reliable and robust synthesis of nucleic acid-containing LbL liposomes. This includes a study of solution conditions, such as $\mathrm{pH}$, ionic strength, salt composition, and valency, and their impact on the preparation of LbL nanoparticles. Our results provide insight into the selection of solution conditions to control the degree of ionization and the electrostatic screening length to suit the adsorption of nucleic acids and synthetic polypeptides. The optimization of these parameters led to a roughly 8-fold improvement in nucleic acid loading in LbL liposomes, indicating the
\end{abstract}

\footnotetext{
"Corresponding Author: hammond@mit.edu.

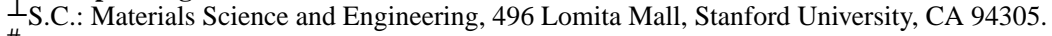

\# These authors contributed equally to this work and are co-first authors. S.C. and N.B. wrote the manuscript. S.C., N.B., and E.D-Y edited the manuscript. S.C. and N.B. designed and carried out all experiments together. E.D-Y. assisted with the data collection and experiment optimization for Figures 6,,, 7, S1, S4, and S6. P.T.H. advised on experimental design, scope, and editing of the final manuscript.

Supporting Information

The Supporting Information is available free of charge on the ACS Publications website at DOI: 10.1021/acsnano.9b00792. Calculations on electrostatic screening lengths and ionic strength, statistics, and supporting figures (the figures include NP stability measurements at varying ionic strengths, charge reversal titrations, gel electrophoresis experiments to determine siRNA retention and stability within LbL NPs, and additional in vitro and in vivo results regarding gene silencing) (PDF)

The authors declare no competing financial interest.
} 
importance of optimizing solution conditions in the preparation of therapeutic LbL nanoparticles. These results highlight the benefits of defining principles for constructing highly effective nanoparticle systems.

\section{Graphical Abstract}

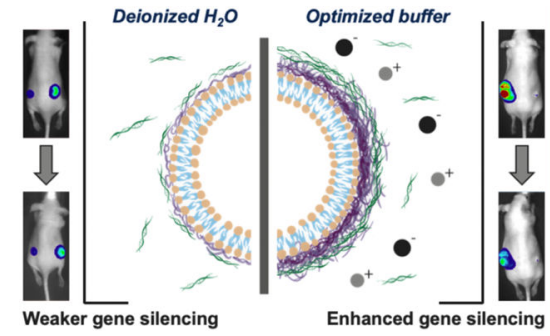

\section{Keywords}

layer-by-layer; self-assembly; nanoparticles; gene delivery; solution conditions; ionic strength; liposomes

Layer-by-layer (LbL) self-assembly is a well-established means for the development of hierarchically organized nanocomposites ${ }^{1-4}$ with numerous applications including drug delivery. ${ }^{5,6}$ Within the realm of biomedical applications, the LbL platform provides the unparalleled ability to incorporate numerous functional materials into discrete nanoparticles (NPs), thereby creating multifunctional drug delivery vehicles. To form LbL NPs, polyelectrolytes of alternating charge are sequentially adsorbed onto an appropriately charged colloidal template. Such templates include NPs with well-documented biomedical applications, such as drug-loaded PLGA ${ }^{7,8}$ and liposomal cores. ${ }^{9,10}$ LbL modification grants these NP cores a multilayered polyelectrolyte film coating, and when the polyelectrolytes in the film are carefully chosen, the resulting LbL NPs gain added functionality beyond that of the core template. Such functionalities include the introduction of stimuli-responsive drug release characteristics, ${ }^{11-13}$ extended systemic circulation times, ${ }^{7,14}$ and active targeting functionality. ${ }^{15}$

One of the most exciting aspects of LbL NPs is the capability to deliver combination therapies in a number of ways. Nucleic acids can be incorporated directly into the LbL film, independent of drugs loaded into the NP core. ${ }^{16}$ This capability allows the delivery of promising therapeutics like small interfering RNA (siRNA) that silence expression of specific genes. When incorporated into an LbL film, siRNA is protected from premature degradation; ${ }^{17}$ furthermore, a carefully selected anionic terminal layer that targets cancer cells allows LbL siRNA carriers to deliver cargo intracellularly. ${ }^{9}$

The clinical potential of LbL NPs is being actively explored by studies that describe increasingly impactful therapeutic formulations. ${ }^{5}$ While this important work continues to identify promising avenues for $\mathrm{LbL}$ nanomedicine, there is an outstanding need to further explore the fundamental parameters that underlie the robust synthesis of nanoscale LbL drug delivery vehicles. The parameter space for the generation of LbL NPs is vast and includes a 
host of variables that include purification methods, mixing conditions, physicochemical properties of the polymer and colloidal species, and adsorption solution conditions. The role of solution conditions on nanoscale LbL self-assembly is especially poorly understood. This deficit translates into obstacles, such as batch-to-batch variability and drug loading limitations, which must be overcome to continue advancing these promising nanomaterials toward the clinic.

Separate batches of LbL NPs can be prepared following the identical sequence of material deposition steps, yet end products can differ from one another, for example, by using different interlayer purification techniques. ${ }^{18,19}$ While it is straightforward to keep purification methods consistent from batch-to-batch, other, subtler, conditions may shift and compromise the consistency of the end product. These conditions include the solution conditions (i.e., aqueous $\mathrm{pH}$ and ionic strength) during self-assembly, which impact the electrostatic forces that both drive LbL assembly and provide colloidal stability to LbL NPs.

Extensive work on macroscale, planar LbL films indicates the importance of solution conditions in mediating efficient LbL assembly, where high concentrations of buffers and salts are routinely used. ${ }^{20}$ In contrast, very limited work has been carried out on solution conditions for colloidal LbL systems, in part due to the sensitivity of electrosterically stabilized colloids to salts. A few studies on colloidal LbL systems have been reported, including work by Caruso and co-workers, ${ }^{21}$ wherein they demonstrated that colloidal gold was layered more efficiently in the presence of sodium chloride $(\mathrm{NaCl})$ but that the particles flocculate at $\mathrm{NaCl}$ concentrations greater than $30 \mathrm{mM}$. Similarly, Decher and colleagues ${ }^{22}$ found that $\mathrm{NaCl}$ could induce thicker film formation on colloidal gold but also cause aggregation at some point between 10 and $100 \mathrm{mM}$ due to electrostatic shielding compromising colloidal suspension stability. On the other hand, Huang et al. ${ }^{23}$ observed that high-aspect-ratio carbon nanotubes could only template LbL assembly at very high salt concentrations $(0.4-1.0 \mathrm{M} \mathrm{NaCl})$. Outside of these systematic studies, the field largely relies on individual reports for insight into synthetic solution conditions, and many reports of LbL colloid syntheses do not explicitly mention solution conditions. For those that do, the conditions can vary significantly: the absence of salt; ${ }^{19,24-26}$ the absence of salt but with a specified $\mathrm{pH}$ (presumably via titration); ${ }^{27-29}$ salt for some layers but not for others; ${ }^{9,30-32}$ salt only during layer deposition but not rinse/purification steps; $;^{22,33-37}$ salt only during interlayer purification; ${ }^{38}$ or salt throughout the synthesis. ${ }^{15,23,39-41}$

Herein, we report a comprehensive study of the solution conditions that influence the synthesis, stability, and characteristics of nucleic acid-containing LbL liposomes. The impact of salts of differing size and valency on the preparation and stability of LbL liposomes was investigated in order to determine criteria for selecting appropriate conditions for stable siRNA incorporation via LbL assembly. Using these optimized conditions, we were able to obtain an 8-fold increase in nucleic acid loading in our LbL NPs, improving the extent and duration of gene silencing in vivo. 


\section{RESULTS AND DISCUSSION}

\section{Layer-by-Layer Assembly Is Highly Sensitive to Solution Conditions.}

Salts and buffers play an invaluable role in the optimization of macroscale LbL modification of planar and microparticle substrates, ${ }^{20,42-45}$ where they provide control over critical factors like the degree of polyelectrolyte ionization, the length scale of electrostatic shielding, and the kinetics and thermodynamics of self-assembly. Increasing salt content is generally understood to facilitate the deposition of thicker layers due to shielding along the polymer backbone. ${ }^{43,46}$ This leads to denser polymer packing in order to achieve the same level of charge compensation. Ionic strength and $\mathrm{pH}$ are also key in nanoscale colloidal $\mathrm{LbL}^{21,22,47}$ and must be carefully weighed against the risks associated with inclusion of salts, such as particle flocculation, film rearrangement, destabilization, and erosion. On the other hand, avoiding salts or buffers greatly limits the parameter space for LbL colloidal systems, leading to less than favorable effects on LbL synthetic outcomes with regard to the amount of material needed to yield a stable, layered particle.

Therefore, in order to tune cargo loading of colloidal LbL assemblies, salt and $\mathrm{pH}$ are important parameters to be considered. In this context, we focused on the effects of salt and $\mathrm{pH}$ on LbL assemblies built upon liposomal substrates, which were chosen due to their versatile properties and applications in the biomedical space. ${ }^{48,49}$ Anionic liposomes comprised of phosphoglycerides, phosphatidylcholines, and cholesterol were chosen for our experiments as substrates for depositing successive layers of polyelectrolytes. ${ }^{50,51}$

We first demonstrated the sensitivity of colloidal $\mathrm{LbL}$ assembly to salt and $\mathrm{pH}$ by comparing the amount of the polycation poly-L-arginine (PLR) required for stable charge conversion ( $\zeta$ $>|30 \mathrm{mV}|, \Delta$ diameter $<100 \mathrm{~nm}$ ) of the anionic liposome substrate in deionized water (DI $\mathrm{H}_{2} \mathrm{O}$, pH 5.5-6.0) and water titrated to $\mathrm{pH} 7.5$ with $20 \mu \mathrm{M} \mathrm{Na}_{2} \mathrm{HPO}_{4}$ (titrated $\mathrm{H}_{2} \mathrm{O}$ ) (Figure 1). We focus on the deposition of PLR because of its well-studied ability to facilitate nucleic acid delivery by facilitating endosomal escape. ${ }^{9}$ To deposit a polyelectrolyte layer onto the liposomal core substrate, an initial polymer titration was performed to identify the optimal polymer weight equivalent (wt. eq.) with respect to the core substrate. At the optimal wt. eq., the substrate charge is successfully converted and the increase in nanoparticle size is small (tens of nanometers by DLS) without requiring vast excess of polyelectrolyte. This study was motivated by our observations that the amount of polyelectrolyte required for stable LbL NP synthesis can vary from day to day, and we attributed this to minor fluctuations in $\mathrm{pH}$ and purity of our $\mathrm{DI}_{2} \mathrm{O}$. We observed that, when the PLR titration was carried out in DI $\mathrm{H}_{2} \mathrm{O}, 0.2$ wt. eq. PLR was required. In contrast, when layering in titrated $\mathrm{H}_{2} \mathrm{O}$, only 0.05 wt. eq. PLR was required for stable charge conversion. Because the $\mathrm{p} K_{\mathrm{a}}$ of PLR side chains is greater than $12^{52}$ and the $\mathrm{p} K_{\mathrm{a}}$ of the phosphoglyceride head groups is less than $3,{ }^{53}$ we believe that differences in ionization states in $\mathrm{DI}_{2} \mathrm{O}$ and titrated $\mathrm{H}_{2} \mathrm{O}$ do not vary substantially and, therefore, cannot explain the observed shifts in PLR required for stable charge conversion. We hypothesized that the presence of salts in the titrated $\mathrm{H}_{2} \mathrm{O}$ affects the amount of polyelectrolyte required for stable charge conversion, potentially through improving the flexibility of polymer chains by shielding charge repulsion along the polycation. However, because the salt concentration is only in the micromolar range in our 
system, it cannot fully shield the charges on the polyelectrolyte components, ${ }^{54}$ which is required for the dense packing of polymers onto the colloidal surface. Therefore, it may be that under dilute salt conditions PLR adsorbs more effciently onto the colloidal template but arranges more sparsely along the surface, thereby requiring less polyelectrolyte mass to achieve charge conversion. Taken together, we believe that thin layers (with less PLR) are forming, which require less polyelectrolyte to achieve charge conversion. These results indicated that altering the concentration of salt could be used to modulate colloidal LbL film properties much as has been done for LbL thin films, specifically the amount of polyelectrolyte contained in each layer. The ability to use ionic strength or $\mathrm{pH}$ as modulators within the window of colloidal stability provides exciting potential for tuning the loading capacity of therapeutic polyelectrolytes such as nucleic acids.

It is important to note that, while micromolar salt concentrations allow charge conversion using 4-fold less PLR, the overall loading of PLR into the film was simultaneously reduced. Being able to reduce the amount of polyelectrolyte required for stable charge conversion can be advantageous when material cost and scale must be considered; however, this can also limit the amount of therapeutic cargo loaded into the colloidal LbL film. Therefore, we were interested in understanding the conditions that lead to higher loading of therapeutic cargo, which we hypothesized also could be tuned using solution conditions during LbL assembly. To investigate how solution conditions influence layering, we performed a screen of a range of different salts and salt concentrations to determine the effects on colloidal LbL selfassembly.

\section{Salt Screen on a Bilayer Nanoparticle System.}

Five salt species were tested at a range of concentrations to determine their impact on the adsorption of PLR onto anionic liposomal cores (Figure 2). In selecting the salts used in this study, we were motivated by prior computational studies ${ }^{55}$ that modeled the role of salt valency and size in the wrapping of nucleic acids on histones and charged spheres. We chose to study the multivalent salts sodium phosphate and sodium citrate, the monovalent salts tris(hydroxymethyl)aminomethane (Tris) hydrochloride and sodium chloride, and the zwitterionic salt HEPES. To start, anionic liposomes (1:1:1 mol ratio DSPC/DSPG/CHOL) were coated with 0.25 wt. eq. $9.6 \mathrm{kDaA}$ PLR with respect to the liposomal core. Solution conditions were varied in this layering step using distinct salt species dissolved in $\mathrm{DI}_{2} \mathrm{O}$ at concentrations ranging from 0.01 to $100 \mathrm{mM}$. Colloidal stability was assessed in each condition by measuring hydrodynamic diameter (left axis, blue curve) and zeta potential (right axis, orange curve) of the resulting LbL particles (Figure 2).

For each of the tested salts, we observed stable size and charge at low salt concentrations, but a sharp increase in particle size and decrease in zeta potential, indicating colloidal aggregation, was observed at high salt concentrations for all conditions except for HEPES. Sodium citrate and sodium phosphate caused flocculation at just 1 and $10 \mathrm{mM}$, respectively (Figure $2 \mathrm{~b}, \mathrm{c}$ ). It is important to note that the observed flocculation (such as the $100 \mathrm{mM}$ sodium citrate and sodium phosphate data points) led to visible aggregates with sizes outside the detectable range for dynamic light scattering (DLS). In comparison to the multivalent species, Tris and sodium chloride did not cause flocculation until $100 \mathrm{mM}$ (Figure 2d,e). 
This difference in stability is likely due to multivalent salts being able to form ionic crosslinks with PLR $^{56}$ that interfere with adsorption and promote aggregation. As noted above, the only exception to the trend was the zwitterionic salt HEPES (Figure 2f), where no significant size destabilization was observed for any of the tested concentrations. Additionally, only at $100 \mathrm{mM}$ HEPES does the zeta potential slightly dip below $30 \mathrm{mV}$.

It has been established that salts can destabilize LbL systems by decreasing the distance over which electrostatic forces dominate (i.e., Debye length), before giving way to attractive forces between particles, causing flocculation. ${ }^{22,57}$ In a stable colloidal system, the Debye length must be long enough to allow for charge-charge repulsion of species in order to avoid aggregation. Because aggregation was observed for all tested salts except HEPES, we hypothesized that HEPES only minimally decreases the Debye length of the system, preventing formation of aggregates. In addition, empirical studies indicated zwitterions, such as HEPES, negligibly contributed to the ionic strength of solution ${ }^{58}$ and, by extension, the electrostatic screening length.

The results of this study confirmed that salt concentration, and thus ionic strength, is an important parameter governing colloidal stability, but these results also revealed that ionic strength alone is insufficient in determining salt compatibility with LbL assembly. Because the Debye length is dependent on ionic strength and varies with the valency and dissociation state of the salt, our observations prompted us to analyze the relationship between the colloidal stability of our particles and the ionic strength of the above salts (Figure S1). For salts like $\mathrm{NaCl}$, we expect a one-to-one correspondence between the concentration of fully dissociated ions in solution and their ionic strength. For the other salts, the relationship between concentration and ionic strength is more complex (see calculations in the Materials and Methods section). If stability trends could be explained solely by ionic strength, we would have expected the data to overlap; however, we only observed partial convergence. This suggests that additional parameters, such as ionic bridging, dissociation state, and counterion size, must be considered for colloidal stability.

\section{Salt Screen on a siLNA-Containing Tetralayer Nanoparticle System.}

The initial model salt screen provided us with the information required to begin understanding the role salt plays in assembling stable LbL NPs. We sought to understand these effects on more complex LbL NP systems used for delivery of therapeutics, such as nucleic acids, specifically a tetralayer LbL NP comprised of a liposomal core (1:1:1 mol ratio of DSPG:DSPC:CHOL); a first layer of PLR; a second layer of a 21-base-pair locked nucleic acid siRNA analogue (siLNA) chosen for its superior stability to degradation by ribonucleases; a third layer of PLR; and a final anionic layer. We chose to synthesize the LbL NP in titrated water up to the fourth layer to eliminate any confounding effects from high salt concentrations and found that siLNA was more stably incorporated compared to layering in $\mathrm{DI}_{2} \mathrm{O}$ (see below). Agarose gel electrophoresis was utilized to track the siLNA content of the LbL NPs throughout the synthesis (Figure 3a). Regarding deposition of the third layer, we observed that incorporation of siLNA appeared to be stabilized with increasing PLR concentration (Figure 3b), in line with our expectations. With increasing amounts of polycation, the PLR layer more effectively shielded siLNA from electrophoresis. 
Specifically, at 0.1 and 0.125 wt. eq. PLR, some siLNA migration into the gel could be seen, but this effect was mitigated at higher weight equivalents. Heparin-treated particles readily released the entrapped siLNA into the gel for all ratios, indicating successful loading of nucleic acid in the particles.

With stable particles (liposome/PLR/siLNA/PLR) in hand, we turned our attention to addition of the fourth layer. In doing so, we also aimed to resolve a challenge particular to LbL films containing polyelectrolyte species that compete for incorporation into the $\mathrm{LbL}$ film, e.g., polyelectrolytes of the same charge but with different charge density, structure, etc. Specifically, we were interested in determining factors to stably retain siLNA in the LbL architecture after the addition of the fourth layer, an anionic polypeptide: propargyl-modified poly-L-aspartic acid (pPLD). In prior experiments, we have observed that addition of the fourth layer in DI $\mathrm{H}_{2} \mathrm{O}$ resulted in displacement of siLNA and accounted for a loss of up to $50 \%$ of the encapsulated cargo (Figure 4a). Furthermore, the extent of siLNA displacement increased with increasing amount of pPLD. We hypothesized that this displacement occurs through competition between $\mathrm{pPLD}$ - a more densely charged and flexible polyelectrolyteand the siLNA - a less charge dense polyelectrolyte described as a stiff, charged rod59-61 which is interpenetrated with the outermost PLR layer. The increased flexibility, and therefore electrostatic pairing, energetically favors incorporation of pPLD over the rigid siLNA, causing the displacement.

Moreover, in addition to minimizing siLNA displacement, when comparing the pPLD titrations carried out in $\mathrm{pH}$ controlled solutions ( $\mathrm{pH}$ 7.0-7.5) and $\mathrm{DI}_{2} \mathrm{O}(\mathrm{pH}$ 5.5-6.0), we noted that, at the higher $\mathrm{pH}$, a lower amount of pPLD (polymer wt. eq. of 2 instead of 4 ) is required to achieve charge conversion of the particle (Figure $4 \mathrm{~d}$ and Figure S3). This suggested that $\mathrm{pH}$ could be used to achieve stable layering at pPLD concentrations that afford high siLNA retention. In this case, the polymer $\left(\mathrm{p} K_{\mathrm{a}}=5\right)^{62}$ is more highly charged at $\mathrm{pH} 7.0-7.5$ than in DI $\mathrm{H}_{2} \mathrm{O}$, and more charge-charge interactions would arise between pPLD and the PLR coating on the LbL NP surface, thus increasing the stability of the layer.

After demonstrating that $\mathrm{pH}$ can be used to control siLNA retention in our colloidal LbL NPs, we investigated the effect of salts, hypothesizing that siLNA-containing LbL NPs synthesized in the presence of salts would be less susceptible to displacement by pPLD due to the generation of thicker, more interpenetrated layers. On the basis of the results from our initial salt screen, we chose to carry out pPLD titrations on the liposome/PLR/siLNA/PLR substrate using the five salts tested previously. Each titration was carried out at $10 \mathrm{mM}$ salt concentration to determine the pPLD amount that would yield stably layered particles while minimizing siLNA loss (Figure 4b). Size and zeta potential characterization indicated that stable particles formed from the highest tested pPLD amount of 4 wt. eq. in all salt solutions except for the multivalent species (Figure 4c,d). This finding was expected given the results of the initial salt screen shown in Figure 2. At 2 wt. eq. pPLD, stable particles were only obtained in Tris and HEPES. Stable particles were not obtained at lower equivalents of pPLD, as the polyelectrolyte amount was not sufficient to achieve stable charge conversion.

While we observed that none of the tested salt conditions were able to eliminate siLNA loss at 4 wt. eq. pPLD, siLNA was displaced to a lesser extent when assembled in salt solutions 
compared to DI $\mathrm{H}_{2} \mathrm{O}$. Minimal siLNA displacement was observed for the stable particles formed in Tris and HEPES solutions at 2 wt. eq. pPLD, supporting our hypothesis that the amount of pPLD is the primary driver of siLNA displacement, and thus when the absolute concentration of pPLD is reduced, we also reduce the opportunity for film rearrangement and loss of siLNA. Moreover, this finding indicated to us that the identity of the salt could be leveraged to achieve charge conversion at a polyelectrolyte concentration that minimizes siLNA displacement.

\section{Tying pH and Salt Together: Finding Optimized Solution Conditions.}

With the observations that buffering to neutral $\mathrm{pH}$ and salt identity allow us to modulate loading capacity and stable incorporation of siLNA in our LbL NPs, we decided to fully synthesize siLNA-containing LbL NPs under optimized solution conditions. To control the $\mathrm{pH}$ of the solution, we decided to use HEPES due to its low ionic strength, its broad buffering range, and the capability to use concentrations as high as $100 \mathrm{mM}$ without impacting colloidal stability. However, HEPES is not effective at shielding electrostatic charge, ${ }^{58}$ and we hypothesized that additional screening would be required to optimally layer siLNA. Using $\mathrm{NaCl}$ as an additive would allow us to finely tune the electrostatics of the system during LbL synthesis. To determine if $\mathrm{pH}$ control alone (i.e., HEPES) was sufficient for efficient particle synthesis and siLNA retention or if additional salt (i.e., $\mathrm{NaCl}$ ) was required for stable film formation, we carried out three separate LbL syntheses: (1) pH 7.5 titrated water (20 $\left.\mu \mathrm{M} \mathrm{Na}_{2} \mathrm{HPO}_{4}\right)$; (2) $25 \mathrm{mM}$ HEPES; and (3) $25 \mathrm{mM}$ HEPES $20 \mathrm{mM}$ $\mathrm{NaCl}$ (Figure 5a-d). Because the role of salt during purification remains an outstanding question for colloidal LbL systems, we sought to establish appropriate conditions for LbL purification in order to determine if salt is beneficial, neutral, or detrimental in the purification step. While removal of salt has been shown to prevent further rearrangement of the LbL film, ${ }^{63}$ there are also empirical reports detailing use of salt only during the purification step. ${ }^{38} \mathrm{~A}$ direct comparison of purification solution conditions has yet to be performed. Therefore, we included a fourth condition where particles layered in the presence of $25 \mathrm{mM}$ HEPES $20 \mathrm{mM} \mathrm{NaCl}$ are buffer exchanged to DI $\mathrm{H}_{2} \mathrm{O}$ during purification via tangential flow filtration (TFF) (Figure 5e,f). ${ }^{19}$ Syntheses were carried out at least three times for each condition.

DLS revealed that LbL NPs prepared in the presence of HEPES/NaCl developed thicker layers, which produced a steady increase in z-average hydrodynamic diameter during the synthesis. Formulations prepared without $\mathrm{NaCl}$ do not exhibit the same steady growth in size. It should be noted that DLS measurements capture the hydrodynamic radius of the particle rather than its true size, complicating interpretation of particle size growth. ${ }^{64}$ For this reason, we primarily focus on the change in size measured by DLS, which we have previously found to correspond to changes in physical size. ${ }^{19}$ All syntheses generated uniform particles with PDI remaining below 0.25, and complete charge conversion was achieved during the addition of different layers. In order to achieve these stable syntheses, the amount of polyelectrolyte was varied significantly with the different solution conditions, again highlighting the ability to modulate the loading capacity of the particles with salt and $\mathrm{pH}$ (Figure 5d). It became clear that LbL NPs synthesized in either titrated water or HEPES alone required much lower equivalents of polyelectrolyte for stable charge conversion and 
had a lower loading capacity of siLNA compared to the particles synthesized in HEPES/ $\mathrm{NaCl}$. This finding validated our hypothesis that shielding is critical for thicker layer deposition and the ability for this vehicle to carry more therapeutic siLNA.

We also directly compared the size and charge of particles purified in salt or in water. Overall, the nanoparticles generated from both methods were of acceptable uniformity, with salt-purified and water-purified particles yielding polydispersity indexes of $0.23 \pm 0.04$ and $0.26 \pm 0.02$, respectively. However, the particles purified in salt began to diverge in size during the final layer. This step yielded much larger particles with a $z$-average hydrodynamic diameter of $245 \pm 7 \mathrm{~nm}$, relative to the $173 \pm 4 \mathrm{~nm}$ diameter of the water-purified system, pointing toward potential destabilization of the particles purified in salt (Figure 5e). Additionally, particles purified in the presence of salt required less polymer in subsequent layers to achieve charge conversion. Therefore, for both conditions, separate weight equivalents of polyelectrolyte were chosen at each layering step in an effort to keep resulting zeta potentials as similar as possible prior to purification (Figure $5 \mathrm{f}$ ). Nonetheless, the need to shift the weight equivalents potentially indicates rearrangement or erosion of the film in the salt-purified system.

When evaluating the encapsulation effciency of the particles synthesized in the four different solution conditions, these values were all found to be near 30\% (Figure 6). However, when examining the weight percent loading of siLNA, we found that particles synthesized with HEPES/ $\mathrm{NaCl}$ carried more siLNA compared to those synthesized in the absence of $\mathrm{NaCl}$. Inclusion of $20 \mathrm{mM} \mathrm{NaCl}$ roughly tripled the average weight percent loading of siLNA from an average loading of $3.7 \pm 3.1 \%$ for particles synthesized in HEPES to $10.5 \pm 3.7 \%$ for particles that are exposed to $\mathrm{NaCl}$ and HEPES throughout the entire synthesis. The positive correlation between flexibility and ionic strength, as exhibited by all polyelectrolytes, allows for a more interpenetrated polyelectrolyte layer via increased electrostatic interactions. We suggest that this effect is primarily seen through the interdigitation of the poly amino acid PLR, as destabilizing salt concentrations are needed to see significant changes in nucleic acid flexibility. We imagine PLR looping with more electrostatic contact points around the stiff siLNA, thereby striking a balance between therapeutic loading and particle stability during syntheses.

Critically, the purification solution conditions allow us to substantially improve siLNA loading of particles layered in HEPES/NaCl. Purifying these particles using HEPES/NaCl yields $10.5 \pm 3.7 \%$ weight loading of siLNA, whereas purifying particles using $\mathrm{DI}_{2} \mathrm{O}$ yields $30.8 \pm 15.7 \%$ loading of siLNA (Figure 6 ). These results demonstrate that, while including $\mathrm{NaCl}$ during the layering stage improved loading of siLNA, purification in the presence of HEPES/ $\mathrm{NaCl}$ had a negative impact on siLNA retention. The introduction of the nanoparticles to millimolar salt concentrations without free siLNA likely leads to conditions in which siLNA is driven to diffuse back into solution. On the other hand, layering in $\mathrm{HEPES} / \mathrm{NaCl}$ and then switching to $\mathrm{DI} \mathrm{H}_{2} \mathrm{O}$ for the subsequent purification step yielded an siLNA weight percent loading of $30.8 \pm 15.7 \%$ - a roughly 8 -fold improvement over HEPES alone. This significant improvement in loading indicates purification in water is advantageous. When LbL NPs are purified via TFF in the presence of $\mathrm{NaCl}$, the salts provide a dynamic environment, potentially causing the $\mathrm{LbL}$ film to rearrange and 
disassemble, resulting in partial loss of adsorbed material. This observation is consistent with a prior study ${ }^{65}$ that demonstrated that planar LbL constructs can be eroded after exposure to continuous flow of polymer and salt.

The increase in siLNA weight percent loading with respect to layer stability was further investigated by incubating tetralayer (liposome/PLR/siLNA/PLR/pPLD) loaded particles overnight in either HEPES/NaCl or water, with and without excess PLD (Figure S4). We hypothesized that, while the presence of salt leads to thicker layers and higher siLNA loading, continued exposure of our LbL NPs to NaCl/HEPES would lead to film rearrangement, resulting in siLNA leaching out of the particle. Indeed, this is what we observed. Incubating particles in salt for $24 \mathrm{~h}$, both with and without excess polyanion, led to siRNA leaching, whereas particles in water exhibited less leaching. Therefore, it is critical to remove salts from LbL NP solutions after layering is complete in order to maintain the integrity of the layer architecture.

\section{Solution Conditions Are Critical for Effective Gene Silencing in a Model of Ovarian Cancer.}

With particle synthesis and purification parameters determined, we aimed to demonstrate that LbL liposomes prepared following our optimized solution condition method (layered in $25 \mathrm{mM} \mathrm{NaCl}$ and $20 \mathrm{mM}$ HEPES, purified in $\mathrm{DI} \mathrm{H}_{2} \mathrm{O}$ ) are able to mediate improved gene silencing. We chose to test the gene delivery efficacy of our optimized LbL NP formulation to the human ovarian cancer cell line OVCAR8 both in vitro and in vivo. The practical consequences for optimizing siLNA loading become apparent in the ability of the final siLNA LbL liposomes to transfect cells. LbL liposomes prepared following the optimized method (layered in $25 \mathrm{mM} \mathrm{NaCl}$ and $20 \mathrm{mM}$ HEPES, purified in $\mathrm{DI}_{2} \mathrm{O}$ ) are able to mediate improved gene silencing compared to particles prepared under suboptimal solution conditions (Figure S5). Luciferized OVCAR8 cells were treated with nanoparticles at a dose of $30 \mathrm{nM}$ siLNA, and luciferase expression was assessed after 1 day. LbL liposomes prepared using HEPES/NaCl conditions showed statistically significant improvement over NPs made in either DI $\mathrm{H}_{2} \mathrm{O}$ or titrated water (expression reduced to $63 \pm 7 \%$ in HEPES/ $\mathrm{NaCl}$ NPs compared to $79 \pm 2$ and $94 \pm 6 \%$ for titrated and $\mathrm{DI} \mathrm{H}_{2} \mathrm{O}$ NPs, respectively).

Because the OVCAR8 cell line can provide a more realistic model of ovarian cancer than prior models, ${ }^{66}$ the ability to transfect this line is of interest for advancing preclinical studies. Consequently, we investigated whether synthetic solution conditions impact LbL liposome transfection efficiency in a subcutaneous flank xenograft of OVCAR8. Following a single tail vein administration of LbL liposomes, we noted prolonged silencing of the target gene luciferase with $\mathrm{LbL}$ liposomes formed using optimal solution conditions relative to those prepared in DI $\mathrm{H}_{2} \mathrm{O}$ (Figure 7). Our prior work showed effective gene silencing using a 1 and $2 \mathrm{mg} / \mathrm{kg}$ dosing of siRNA; 9,67 therefore, in order to demonstrate improved carrier stability, we tested a lower siLNA dose of $0.5 \mathrm{mg} / \mathrm{kg}$. After a single $0.5 \mathrm{mg}$ of siLNA $/ \mathrm{kg}$ injection, the nanoparticle prepared in $\mathrm{DI} \mathrm{H}_{2} \mathrm{O}$ mediated knockdown of luciferase signal 1 day after treatment $(88.6 \pm 6.8 \%$ expression relative to pretreatment) and dissipated 2 days after treatment. On the other hand, the gene silencing effect of the solution-conditionoptimized formulation improved out to 3 days post-treatment, with luciferase expression (relative to pretreatment) decreasing from $84.6 \pm 17.3 \%$ at day 1 , to $70.9 \pm 15.8 \%$ at day 2 , 
and finally to $68.5 \pm 6.0 \%$ at day 3 . Notably, at day 2 (Figure 7c), two points deviated from the mean for the nanoparticles made in HEPES and $\mathrm{NaCl}$, but by day 3 , luciferase expression dipped back down in line with the other subjects (Figure S6). The data demonstrate the ability to mediate prolonged silencing following a single dose at $0.5 \mathrm{mg} / \mathrm{kg}$ in this ovarian cancer model. The results also indicate that $\mathrm{LbL}$ assembly conditions can have a significant impact on the bioactivity of LbL drug delivery vehicles both in vitro and in vivo.

\section{CONCLUSIONS}

These results demonstrate the importance of carefully optimizing solution conditions for the self-assembly of LbL nanoparticles, especially for systems designed for drug delivery. Consideration of the various molecular forces and the physical processes that mediate efficient layering can be used to design solution conditions to optimize the loading of functional biopolymers into this emerging class of nanocarrier. Notably, we report a robust approach to functionalize a critical class of drug delivery vehicles, liposomes, with LbL films to expand their biomedical functionality. Optimal film formation on these drug carriers required tuning solution conditions during both the layer deposition and purification stages of the LbL film assembly process. This work offers a useful template for optimization of similar systems and aims to promote greater investigation into the role of process conditions in the development of these systems.

\section{MATERIALS AND METHODS}

$\mathrm{NaCl}$ powder was purchased from Macron Fine Chemicals. One $\mathrm{M}$ Tris- $\mathrm{HCl}$ solution was purchased from Sigma-Aldrich. HEPES, sodium citrate, $\mathrm{Na}_{2} \mathrm{HPO}_{4}$, and $\mathrm{NaH}_{2} \mathrm{PO}_{4}$ powders were purchased from MilliporeSigma. 1,2-Distearoyl-sn-glycero-3-phosphocholine (DSPC), 1-palmitoyl-2-oleoyl-sn-glycero-3-phospho-( $1^{\prime}$-rac-glycerol) (sodium salt) (POPG), 1,2distearoyl-sn-glycero-3-phospho-( $1^{\prime}$-rac-glycerol) (sodium salt) (DSPG), and cholesterol were purchased from Avanti. Chloroform and methanol were purchased from TCI and Sigma, respectively. Whatman nuclepore polycarbonate hydrophilic membranes $(400,200$, 100 , and $50 \mathrm{~nm}$ sizes) were purchased from GE. All glassware was obtained from Chemglass. 50/15 mL Falcon tubes and 50/5/2 mL DNA loBind Eppendorf tubes were purchased from VWR. Polysytrene latex microspheres (Fluospheres), locked nucleic acid siRNAs (Silencer Select), $5 \mathrm{M}$ bioreagent grade $\mathrm{NaCl}$ solution, and precast EX $2 \%$ agarose E-gels were obtained from Thermo Fisher Scientific. One M bioreagent-grade HEPES was purchased from Fisher Scientific. D02-E100-05-N and C02-E100-05-N tangential flow filtration filters were purchased from Spectrum Laboratories. Poly(L-arginine hydrochloride) (9.6 kDa, $38.5 \mathrm{kDa})$, poly(L-glutamic acid sodium salt) $(15 \mathrm{kDa})$, and poly(L-aspartic acid sodium salt) $(14 \mathrm{kDa})$ were purchased from Alamanda Polymers. Dextran sulfate sodium salt (15 kDa) was purchased from MilliporeSigma. Hyaluronic acid (20 kDa) was purchased from LifeCore. Heparin sulfate was a generous gift from the Sasisekharan Lab. Propargylmodified PLD was synthesized according to prior methods ${ }^{68,69}$ Polystyrene semimicro cuvettes for the Malvern Zetasizer were purchased from VWR, and DTS1070 folded capillary cells were purchased directly from Malvern. Black 364-well plates for the Wyatt DLS were purchased directly from the Peterson (1957) Nanotechnology Core Facility. 
The OVCAR8 human ovarian cancer cell line was a gift from the Bhatia Lab at MIT. Tissue culture plasticware (T75, T25, clear and white 96-well plates), trypsin EDTA, penicillin streptomycin, and RPMI 1640 media were purchased from Corning. Fetal bovine serum was purchased from Gibco. Steady-Glo luciferase expression assay and CellTiter-Fluor viability assay were purchased from Promega.

\section{Preparation of Liposomal Cores.}

DSPC, POPG, and cholesterol were dissolved in chloroform, and DSPG was dissolved in a 65:35:8 mixture of chloroform, methanol, and deionized water (Milli-Q). Lipid mixtures composed of $66.7 \mathrm{~mol} \%$ phospholipid and 33.3\% cholesterol were prepared in roundbottom flasks (RBFs) (10 or $50 \mathrm{~mL}$ depending on scale), and for DSPG formulations, methanol was added dropwise until the solution cleared. The lipid solution was evaporated using a BUCHI RotoVap system under heat $\left(60^{\circ} \mathrm{C}\right.$, water bath) until completely dry $(<15$ mbar). A Branson sonicator bath was filled with reverse-osmosis water and heated until $>65^{\circ} \mathrm{C}$, at which point the RBF with the lipid film was partially submerged in the bath and a volume of $\mathrm{dH}_{2} \mathrm{O}$ was added to resuspend the lipid film to a $1 \mathrm{mg}$ of $\mathrm{lipid} / \mathrm{mL}$ solution. The liposome solution was sonicated for roughly $1 \mathrm{~min}$ and then transferred to an Avestin LiposoFast LF-50 liposome extruder. The extruder was connected to a Cole-Parmer Polystat Heated Recirculator Bath to maintain a temperature $>65^{\circ} \mathrm{C}$. The liposome solution was extruded through successively smaller nuclepore membranes until a 50-100 nm liposome was obtained. Generally, this required one pass through a stack of two to three 400,200, 100 , and $50 \mathrm{~nm}$ membranes. Liposomes were characterized for size and zeta using the techniques outlined below.

\section{Buffer Preparation.}

For the initial PLR layering screen, all buffers were prepared as $200 \mathrm{mM}$ stock solutions in Milli-Q water and serially diluted to generate $2 \times$ strength solutions for each concentration to be tested. Solution $\mathrm{pH}$ was measured using both $\mathrm{pH}$ paper and a Hanna electronic $\mathrm{pH}-$ meter. Tris- $\mathrm{HCl}$ and sodium citrate were titrated with $\mathrm{HCl}$ to obtain $\mathrm{pH}$ 7-7.6. Sodium phosphate was titrated with $\mathrm{NaOH}$ to $\mathrm{pH} 7-7.6$. For the $\mathrm{pH}$-sensitivity experiment in Figure 1, DI H2O was titrated to $\mathrm{pH} 7.5$ with $\mathrm{Na}_{2} \mathrm{HPO}_{4}$. For experiments using the optimized solution conditions, a $50 \mathrm{mM}$ HEPES $40 \mathrm{mM} \mathrm{NaCl}$ solution was prepared in Milli-Q water and titrated to $\mathrm{pH} 7.5$.

\section{Layer-by-Layer Assembly.}

Nanoparticles were layered by adding an equal volume of nanoparticle solution (not exceeding $1 \mathrm{mg} / \mathrm{mL}$ ) to an equal volume of polyelectrolyte solution under sonication (Branson bath sonicator, room temperature). The mixture was sonicated for roughly $5 \mathrm{~s}$. The optimal wt. eq. for each layer was determined prior to the deposition step via a polyelectrolyte titration using 25-50 uL samples of the nanoparticle solution for each tested wt. eq. The test ratios were mixed as above but only incubated for 5-10 min before characterization. If the resulting particle had a zeta potential greater than $30 \mathrm{mV}$ (either positive or negative) and an appropriate size, it was chosen as the optimal ratio. 
For conditions involving a buffer or salt throughout the entire synthesis, the polyelectrolyte solution is prepared in $2 \times$ strength buffer for the initial layer and then in $1 \times$ strength buffer for subsequent layers. For conditions that remove the salt during purification steps, the polyelectrolyte solution is prepared in $2 \times$ strength buffer for all deposition steps. Bioreagentgrade $\mathrm{NaCl}$ and HEPES were used for syntheses involving siRNA. DNA loBind tubes are used as the mixing vessels for all experiments, to prevent nonspecific adsorption of siRNA and other polymers to the plasticware.

The layered particle is allowed to incubate at room temperature for $1 \mathrm{~h}$ and is then purified using the tangential flow filtration method, as described previously. ${ }^{19}$ Briefly, crude nanoparticle solution is connected to a Spectrum Laboratories KrosFlo II system using masterflex, Teflon-coated tubing. D02-E100-05-N (batch volume $>5 \mathrm{~mL}$ ) or C02-E100-05$\mathrm{N}$ (batch volume $<1 \mathrm{~mL}$ ) $100 \mathrm{kDa}$ filters were used to purify the particles until 5 volumeequivalents were collected in the permeate. For cationic layers, the TFF filter was pretreated with a solution of free polycation (same concentration used for layering) in order to minimize nonspecific adsorption of particles to the membrane walls. Samples were run at 80 $\mathrm{mL} / \mathrm{min}$ (size 16 tubing, used with D02-series filters) or $13 \mathrm{~mL} / \mathrm{min}$ (size 13 tubing, used with $\mathrm{C} 02$-series filters). Once pure, the sample was either concentrated (by disconnecting the buffer reservoir) or recovered via reversing the direction of the peristaltic pump. For more complete yields, $1-3 \mathrm{~mL}$ of the appropriate buffer was run backward through the tubing to recover any remaining particles. This process was repeated until the desired LbL formulation was obtained. Exchange buffer was chosen to be consistent with the intended synthetic solution conditions.

\section{Characterization of LbL Nanoparticles.}

Nanoparticles were characterized using dynamic light scattering. The Wyatt Dyna Pro was used for high throughput experiments shown in Figures 3 and 4 and the Malvern ZS90 Particle Analyzer for all other size measurements reported. Zeta potential measurements were made using laser Doppler electrophoresis on the Malvern ZS90. Samples were diluted in Milli-Q water for all measurements. Results from the Malvern are reported using the SD of three measurements.

\section{Determination of siRNA Loading and Retention (Agarose Gel and NanoDrop).}

Agarose gels were used to determine the complexation state and loading of siRNA. To determine complexation state, nanoparticles were diluted and $20 \mu \mathrm{L}$ of the diluted particles were added to the wells of a precast EX 2\% agarose E-gel. To determine loading, the LbL nanoparticles were dissociated by addition of excess heparin; e.g., the particles were diluted in a solution of $5 \mathrm{mg} / \mathrm{mL}$ heparin in $\mathrm{H}_{2} \mathrm{O}$ and heated $\left(37^{\circ} \mathrm{C}\right)$ for $15 \mathrm{~min}$. The gel was run using the E-gel system (Thermo Fisher) for $5 \mathrm{~min}$ and then visualized using a Typhoon laser scanning imager (GE).

To quantify encapsulation efficiency, permeate fractions were collected during tangential flow filtration of the siRNA-coated nanoparticles. Fractions were analyzed for siRNA concentration using the NanoDrop system (Thermo Fisher) and used to determine the total amount of siRNA removed from the system. The encapsulation efficiency was used in 
conjunction with the known amount of siRNA introduced into the system and the yieldadjusted $\mathrm{mg}$ of lipid in the system to determine weight percent loading.

\section{Cell Culture and Transfection.}

OVCAR8 cells were seeded at a density of 20,000 cells per well in a 96-well plate in complete RPMI 1640 media (10\% FBS, 1\% pen-strep). Cells were allowed to adhere to the plate for $24 \mathrm{~h}$ prior to treatment with nanoparticles. Nanoparticles were dosed to provide equivalent siRNA concentrations across conditions $(30 \mathrm{nM})$. After $24 \mathrm{~h}$, cell viability and luciferase expression were measured using the multiplexed CellTiter-Fluor and Steady-Glo assay kits according to the manufacturer's protocols. Luciferase expression was normalized to the well viability and then normalized to expression from untreated cells.

\section{In Vivo Transfection.}

Female NCR nu/nu mice were purchased from Taconic and subcutaneously injected with mCherry/Luciferase expressing OVCAR8 cells ( 1 million cells per flank, $200 \mu \mathrm{L}$ injections in 1:1 PBS:MatriGel). Tumors were allowed to establish over the course of 1 month and then randomized into treatment groups. Mice were then injected (tail vein injection, $200 \mu \mathrm{L}$ bolus) with nanoparticles to provide a $0.5 \mathrm{mg} / \mathrm{kg}$ dose of siRNA against the luciferase gene. Whole-animal imaging was performed using the Xenogen IVIS Imaging System (PerkinElmer) following an intraperitoneal injection of d-luciferin ( $200 \mu \mathrm{L}, 15 \mathrm{mg} / \mathrm{mL}, 10$ min incubation prior to imaging). These experiments were approved by the Massachusetts Institute of Technology Committee on Animal Care (CAC).

\section{Supplementary Material}

Refer to Web version on PubMed Central for supplementary material.

\section{ACKNOWLEDGMENTS}

This work was supported by the Department of Defense's Ovarian Cancer Research Program (W81XWH-13-1-0151) and a grant from the Koch Institute's Marble Center for Cancer Nanomedicine. The authors also thank the MIT Koch Institute Swanson Biotechnology Center, which is supported by the Koch Institute Core Grant P30-CA14051 from the NCI, for the use of facilities and specifically the Peterson (1957) Nanotechnology Core Facility. This material is based on work made possible by the Sloan UCEM Fellowship and Siebel Scholars Fellowship for S.C. and a fellowship from the Koch Institute's Marble Center for Cancer Nanomedicine for N.B. The authors would like to thank Dr. Monica Olvera de la Cruz, Dr. Alfredo Alexander-Katz, and Stephanie Kong for helpful discussion and advice during the preparation of this work.

\section{REFERENCES}

(1). Lvov Y; Decher G; Mohwald H Assembly, Structural Characterization, and Thermal-Behavior of Layer-by-Layer Deposited Ultrathin Films of Poly(Vinyl Sulfate) and Poly(Allylamine). Langmuir 1993, 9, 481-486.

(2). Hammond PT Form and Function in Multilayer Assembly: New Applications at the Nanoscale. Adv. Mater 2004, 16, 1271-1293.

(3). Iler RK Multilayers of Colloidal Particles. J. Colloid Interface Sci. 1966, 21, 569-594.

(4). Wang Y; Angelatos AS; Caruso F Template Synthesis of Nanostructured Materials via Layer-byLayer Assembly. Chem. Mater 2008, 20, 848-858.

(5). Correa S; Dreaden EC; Gu L; Hammond PT Engineering Nanolayered Particles for Modular Drug Delivery. J. Controlled Release 2016, 240, 364-386. 
(6). Johnston APR; Cortez C; Angelatos AS; Caruso F Layer-by-Layer Engineered Capsules and Their Applications. Curr. Opin. Colloid Interface Sci. 2006, 11, 203-209.

(7). Morton SW; Poon Z; Hammond PT The Architecture and Biological Performance of Drug-Loaded LbL Nanoparticles. Bio-materials 2013, 34, 5328-5335.

(8). Luo R; Neu B; Venkatraman SS Surface Functionalization of Nanoparticles to Control Cell Interactions and Drug Release. Small 2012, 8, 2585-2594. [PubMed: 22674655]

(9). Deng ZJ; Morton SW; Ben-Akiva E; Dreaden EC; Shopsowitz KE; Hammond PT Layer-by-Layer Nanoparticles for Systemic Codelivery of an Anticancer Drug and siRNA for Potential TripleNegative Breast Cancer Treatment. ACS Nano 2013, 7, 9571-9584. [PubMed: 24144228]

(10). Dreaden EC; Kong YW; Morton SW; Correa S; Choi KY; Shopsowitz KE; Renggli K; Drapkin R; Yaffe MB; Hammond PT Tumor-Targeted Synergistic Blockade of MAPK and PI3K from a Layer-by-Layer Nanoparticle. Clin. Cancer Res. 2015, 21, 4410-4419. [PubMed: 26034127]

(11). Li P; Liu D; Miao L; Liu C; Sun X; Liu Y; Zhang N A pH-Sensitive Multifunctional Gene Carrier Assembled via Layer-by-Layer Technique for Efficient Gene Delivery. Int. J. Nanomed 2012, 7, 925-939.

(12). Yang XZ; Du JZ; Dou S; Mao CQ; Long HY; Wang J Sheddable Ternary Nanoparticles for Tumor Acidity-Targeted siRNA Delivery. ACS Nano 2012, 6, 771-781. [PubMed: 22136582]

(13). Ye CH; Drachuk I; Calabrese R; Dai HQ; Kaplan DL; Tsukruk VV Permeability and Micromechanical Properties of Silk Ionomer Microcapsules. Langmuir 2012, 28, 12235-12244. [PubMed: 22834790]

(14). Ramasamy T; Haidar ZS; Tran TH; Choi JY; Jeong JH; Shin BS; Choi HG; Yong CS; Kim JO Layer-by-Layer Assembly of Liposomal Nanoparticles with PEGylated Polyelectrolytes Enhances Systemic Delivery of Multiple Anticancer Drugs. Acta Biomater. 2014, 10, 51165127. [PubMed: 25169256]

(15). Dreaden EC; Morton SW; Shopsowitz KE; Choi JH; Deng ZJ; Cho NJ; Hammond PT Bimodal Tumor-Targeting from Microenvironment Responsive Hyaluronan Layer-by-Layer (LbL) Nanoparticles. ACS Nano 2014, 8, 8374-8382. [PubMed: 25100313]

(16). Gu L; Deng ZJ; Roy S; Hammond PT A Combination RNAi-Chemotherapy Layer-by-Layer Nanoparticle for Systemic Targeting of KRAS/P53 with Cisplatin to Treat Non-Small Cell Lung Cancer. Clin. Cancer Res. 2017, 23, 7312-7323. [PubMed: 28912139]

(17). Yao Y; Su Z; Liang Y; Zhang N pH-Sensitive Carboxymethyl Chitosan-Modified Cationic Liposomes for Sorafenib and siRNA Co-Delivery. Int. J. Nanomed 2015, 10, 6185-6197.

(18). Richardson JJ; Ejima H; Lorcher SL; Liang K; Senn P; Cui J; Caruso F Preparation of Nano- and Microcapsules by Electrophoretic Polymer Assembly. Angew. Chem., Int. Ed 2013, 52, 64556458.

(19). Correa S; Choi KY; Dreaden EC; Renggli K; Shi A; Gu L; Shopsowitz KE; Quadir MA; BenAkiva E; Hammond PT Highly Scalable, Closed-Loop Synthesis of Drug-Loaded, Layer-byLayer Nanoparticles. Adv. Funct. Mater 2016, 26, 991-1003. [PubMed: 27134622]

(20). Bertrand P; Jonas A; Laschewsky A; Legras R Ultrathin Polymer Coatings by Complexation of Polyelectrolytes at Interfaces: Suitable Materials, Structure and Properties. Macromol. Rapid Commun. 2000, 21, 319-348.

(21). Gittins DI; Caruso F Tailoring the Polyelectrolyte Coating of Metal Nanoparticles. J. Phys. Chem. B 2001, 105, 6846-6852.

(22). Schneider G; Decher G Functional Core/Shell Nanoparticles via Layer-by-Layer Assembly. Investigation of the Experimental Parameters for Controlling Particle Aggregation and for Enhancing Dispersion Stability. Langmuir 2008, 24, 1778-1789. [PubMed: 18225923]

(23). Huang SC; Artyukhin AB; Wang Y; Ju JW; Stroeve P; Noy A Persistence Length Control of the Polyelectrolyte Layer-by-Layer Self-Assembly on Carbon Nanotubes. J. Am. Chem. Soc 2005, 127, 14176-14177. [PubMed: 16218599]

(24). Zhang P; Chiu YC; Tostanoski LH; Jewell CM Polyelectrolyte Multilayers Assembled Entirely from Immune Signals on Gold Nanoparticle Templates Promote Antigen-Specific T Cell Response. ACS Nano 2015, 9, 6465-6477. [PubMed: 26035231]

(25). Schneider G; Decher G From Functional Core/Shell Nanoparticles Prepared via Layer-by-Layer Deposition to Empty Nanospheres. Nano Lett. 2004, 4, 1833-1839. 
(26). Lee SK; Tung CH A Fabricated siRNA Nanoparticle for Ultra-Long Gene Silencing In Vivo. Adv. Funct. Mater 2013, 23, 3488-3493. [PubMed: 24999314]

(27). Poon Z; Lee JB; Morton SW; Hammond PT Controlling In Vivo Stability and Biodistribution in Electrostatically Assembled Nanoparticles for Systemic Delivery. Nano Lett. 2011, 11, 20962103. [PubMed: 21524115]

(28). Cui W; Wang A; Zhao J; Yang X; Cai P; Li J Layer by Layer Assembly of Albumin Nanoparticles with Selective Recognition of Tumor Necrosis Factor-Related Apoptosis-Inducing Ligand (TRAIL). J. Colloid Interface Sci. 2016, 465, 11-17. [PubMed: 26641559]

(29). Shutava TG; Balkundi SS; Vangala P; Steffan JJ; Bigelow RL; Cardelli JA; O’Neal DP; Lvov YM Layer-by-Layer-Coated Gelatin Nanoparticles as a Vehicle for Delivery of Natural Polyphenols. ACS Nano 2009, 3, 1877-1885. [PubMed: 19534472]

(30). Bishop CJ; Tzeng SY; Green JJ Degradable Polymer-Coated Gold Nanoparticles for Co-Delivery of DNA and siRNA. Acta Biomater. 2015, 11, 393-403. [PubMed: 25246314]

(31). Guo S; Huang Y; Jiang Q; Sun Y; Deng L; Liang Z; Du Q; Xing J; Zhao Y; Wang PC; Dong A; Liang XJ Enhanced Gene Delivery and siRNA Silencing by Gold Nanoparticles Coated with Charge-Reversal Polyelectrolyte. ACS Nano 2010, 4, 5505-5511. [PubMed: 20707386]

(32). Han L; Zhao J; Zhang X; Cao W; Hu X; Zou G; Duan X; Liang XJ Enhanced siRNA Delivery and Silencing Gold-Chitosan Nanosystem with Surface Charge-Reversal Polymer Assembly and Good Biocompatibility. ACS Nano 2012, 6, 7340-7351. [PubMed: 22838646]

(33). Son KJ; Yoon HJ; Kim JH; Jang WD; Lee Y; Koh WG Photosensitizing Hollow Nanocapsules for Combination Cancer Therapy. Angew. Chem., Int. Ed 2011, 50, 11968-11971.

(34). Shen J; Kim HC; Mu C; Gentile E; Mai J; Wolfram J; Ji LN; Ferrari M; Mao ZW; Shen H Multifunctional Gold Nanorods for siRNA Gene Silencing and Photothermal Therapy. Adv. Healthcare Mater. 2014, 3, 1629-1637.

(35). Caruso RA; Susha A; Caruso F Multilayered Titania, Silica, and Laponite Nanoparticle Coatings on Polystyrene Colloidal Templates and Resulting Inorganic Hollow Spheres. Chem. Mater 2001, 13, 400-409.

(36). Burke SE; Barrett CJ Acid-Base Equilibria of Weak Polyelectrolytes in Multilayer Thin Films. Langmuir 2003, 19, 3297-3303.

(37). Melby ES; Lohse SE; Park JE; Vartanian AM; Putans RA; Abbott HB; Hamers RJ; Murphy CJ; Pedersen JA Cascading Effects of Nanoparticle Coatings: Surface Functionalization Dictates the Assemblage of Complexed Proteins and Subsequent Interaction with Model Cell Membranes. ACS Nano 2017, 11, 5489-5499. [PubMed: 28482159]

(38). Elbakry A; Zaky A; Liebkl R; Rachel R; Goepferich A; Breunig M Layer-by-Layer Assembled Gold Nanoparticles for siRNA Delivery. Nano Lett. 2009, 9, 2059-2064. [PubMed: 19331425]

(39). Mayya KS; Schoeler B; Caruso F Preparation and Organization of Nanoscale PolyelectrolyteCoated Gold Nanoparticles. Adv. Funct. Mater 2003, 13, 183-188.

(40). Lohse SE; Eller JR; Sivapalan ST; Plews MR; Murphy CJ A Simple Millifluidic Benchtop Reactor System for the High-Throughput Synthesis and Functionalization of Gold Nanoparticles with Different Sizes and Shapes. ACS Nano 2013, 7, 4135-4150. [PubMed: 23634842]

(41). Chen Z; Zhang L; He Y; Shen Y; Li Y Enhanced shRNA Delivery and ABCG2 Silencing by Charge-Reversible Layered Nanocarriers. Small 2015, 11, 952-962. [PubMed: 25330768]

(42). Ferreira M; Rubner MF Molecular-Level Processing of Conjugated Polymers 0.1. Layer-byLayer Manipulation of Conjugated Polyions. Macromolecules 1995, 28, 7107-7114.

(43). Clark SL; Montague MF; Hammond PT Ionic Effects of Sodium Chloride on the Templated Deposition of Polyelectrolytes Using Layer-by-Layer Ionic Assembly. Macromolecules 1997, 30, 7237-7244.

(44). Lee L; Cavalieri F; Johnston APR; Caruso F Influence of Salt Concentration on the Assembly of DNA Multilayer Films. Langmuir 2010, 26, 3415-3422. [PubMed: 19891451]

(45). Jiang CY; Tsukruk VV Freestanding Nanostructures via Layer-by-Layer Assembly. Adv. Mater 2006, 18, 829-840.

(46). Lvov Y; Ariga K; Onda M; Ichinose I; Kunitake T Alternate Assembly of Ordered Multilayers of $\mathrm{SiO} 2$ and Other Nanoparticles and Polyions. Langmuir 1997, 13, 6195-6203. 
(47). Schneider GF; Decher G From "Nano-Bags" to "Micro-Pouches". Understanding and Tweaking Flocculation-based Processes for the Preparation of New Nanoparticle-Composites. Nano Lett. 2008, 8, 3598-3604. [PubMed: 18850753]

(48). Van Norman GA Drugs, Devices, and the FDA: Part 1 An Overview of Approval Processes for Drugs. JACC Basic Transl. Sci 2016, 1, 170-179. [PubMed: 30167510]

(49). Bobo D; Robinson KJ; Islam J; Thurecht KJ; Corrie SR Nanoparticle-Based Medicines: A Review of FDA-Approved Materials and Clinical Trials to Date. Pharm. Res 2016, 33, 2373 2387. [PubMed: 27299311]

(50). Sercombe L; Veerati T; Moheimani F; Wu SY; Sood AK; Hua S Advances and Challenges of Liposome Assisted Drug Delivery. Front. Pharmacol 2015, 6, 1-13. [PubMed: 25805991]

(51). Briuglia ML; Rotella C; McFarlane A; Lamprou DA Influence of Cholesterol on Liposome Stability and on In Vitro Drug Release. Drug Delivery Transl. Res 2015, 5, 231-242.

(52). Fitch CA; Platzer G; Okon M; Garcia-Moreno B; McIntosh LP Arginine: Its pK(a) Value Revisited. Protein Sci. 2015, 24, 752-761. [PubMed: 25808204]

(53). Marsh D CRC Handbook of Lipid Bilayers; CRC Press: Boca Raton, FL, 1990.

(54). Carrillo JMY; Dobrynin AV Polyelectrolytes in Salt Solutions: Molecular Dynamics Simulations. Macromolecules 2011, 44, 5798-5816.

(55). Carnal F; Stoll S Adsorption of Weak Polyelectrolytes on Charged Nanoparticles. Impact of Salt Valency, pH, and Nanoparticle Charge Density. Monte Carlo Simulations. J. Phys. Chem. B 2011, 115, 12007-12018. [PubMed: 21902229]

(56). Mogaki R; Hashim PK; Okuro K; Aida T Guanidinium-Based "Molecular Glues" for Modulation of Biomolecular Functions. Chem. Soc. Rev 2017, 46, 6480-6491. [PubMed: 29034942]

(57). Huang SCJ; Artyukhin AB; Wang YM; Ju JW; Stroeve P; Noy A Persistence Length Control of the Polyelectrolyte Layer-by-Layer Self-Assembly on Carbon Nanotubes. J. Am. Chem. Soc 2005, 127, 14176-14177. [PubMed: 16218599]

(58). Stellwagen E; Prantner JD; Stellwagen NC Do Zwitterions Contribute to the Ionic Strength of a Solution? Anal. Biochem 2008, 373, 407-409. [PubMed: 18022379]

(59). Hagerman PJ Flexibility of RNA. Annu. Rev. Biophys. Biomol. Struct 1997, 26, 139-156. [PubMed: 9241416]

(60). Kebbekus P; Draper DE; Hagerman P Persistence Length of RNA. Biochemistry 1995, 34, 43544357. [PubMed: 7535562]

(61). Gary DJ; Puri N; Won YY Polymer-Based siRNA Delivery: Perspectives on the Fundamental and Phenomenological Distinctions from Polymer-Based DNA Delivery. J. Controlled Release 2007, 121, 64-73.

(62). Nemeth C; Gyarmati B; Abdullin T; Laszlo K; Szilagyi A Poly(Aspartic Acid) with Adjustable pH-Dependent Solubility. Acta Biomater. 2017, 49, 486-494. [PubMed: 27915021]

(63). Parveen N; Schonhoff M Swelling and Stability of Polyelectrolyte Multilayers in Ionic Liquid Solutions. Macromolecules 2013, 46, 7880-7888.

(64). Sukhorukov GB; Donath E; Lichtenfeld H; Knippel E; Knippel M; Budde A; Möhwald H Layerby-Layer Self Assembly of Polyelectrolytes on Colloidal Particles. Colloids Surf., A 1998, 137, 253-266.

(65). Kovacevic D; van der Burgh S; de Keizer A; Stuart MAC Kinetics of Formation and Dissolution of Weak Polyelectrolyte Multilayers: Role of Salt and Free Polyions. Langmuir 2002, 18, 56075612.

(66). Mitra AK; Davis DA; Tomar S; Roy L; Gurler H; Xie J; Lantvit DD; Cardenas H; Fang F; Liu YY; Loughran E; Yang J; Stack MS; Emerson RE; Dahl KDC; Barbolina MV; Nephew KP; Matei D; Burdette JE In Vivo Tumor Growth of High-Grade Serous Ovarian Cancer Cell Lines. Gynecol. Oncol 2015, 138, 372-377. [PubMed: 26050922]

(67). Gu L; Deng ZJ; Roy S; Hammond PT A Combination RNAi-Chemotherapy Layer-by-Layer Nanoparticle for Systemic Targeting of KRAS/P53 with Cisplatin to Treat Non-Small Cell Lung Cancer. Clin. Cancer Res. 2017, 23, 7312-7323. [PubMed: 28912139]

(68). Meng FF; Wang JP; Ping QN; Yeo Y Quantitative Assessment of Nanoparticle Biodistribution by Fluorescence Imaging, Revisited. ACS Nano 2018, 12, 6458-6468. [PubMed: 29920064] 
(69). Li J; Wang W; He Y; Li Y; Yan EZ; Zhang K; Irvine DJ; Hammond PT Structurally Programmed Assembly of Translation Initiation Nanoplex for Superior mRNA Delivery. ACS Nano 2017, 11, 2531-2544. [PubMed: 28157292] 
Solution conditions influence layering efficiency

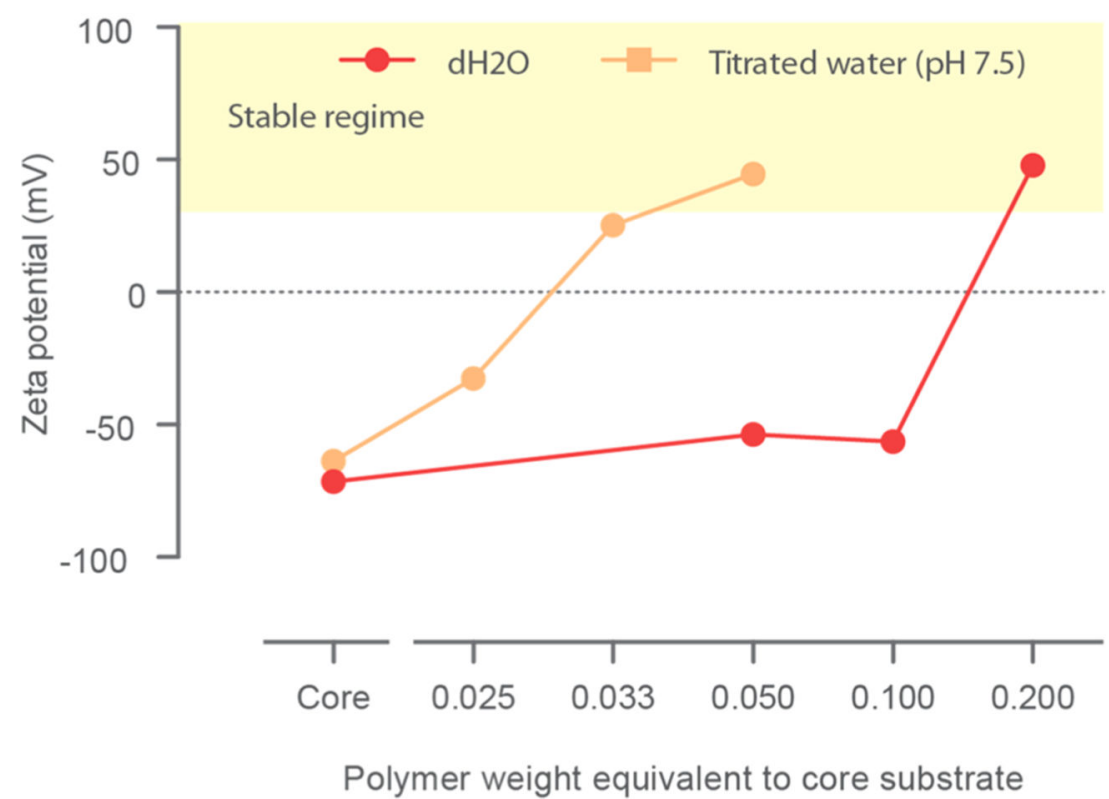

Figure 1.

Anionic liposomes (58:18:24 mol ratio DSPC:POPG:-CHOL) were mixed with PLR at various weight equivalents to identify ratios that yield charge conversion within the stable regime. Depending on if the titration is carried out in $\mathrm{dH}_{2} \mathrm{O}$ or $\mathrm{pH} 7.5$ titrated water $(20 \mu \mathrm{M}$ $\mathrm{Na}_{2} \mathrm{HPO}_{4}$ ), the optimal weight equivalent varies 4-fold-from 0.050 in $20 \mu \mathrm{M} \mathrm{Na}_{2} \mathrm{HPO}_{4}$ or 0.200 in DI $\mathrm{H}_{2} \mathrm{O}$. 

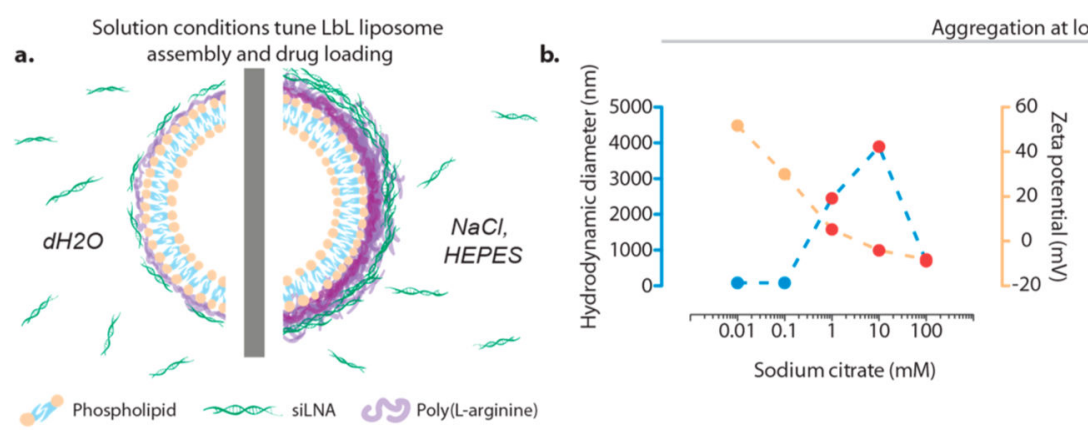

c.
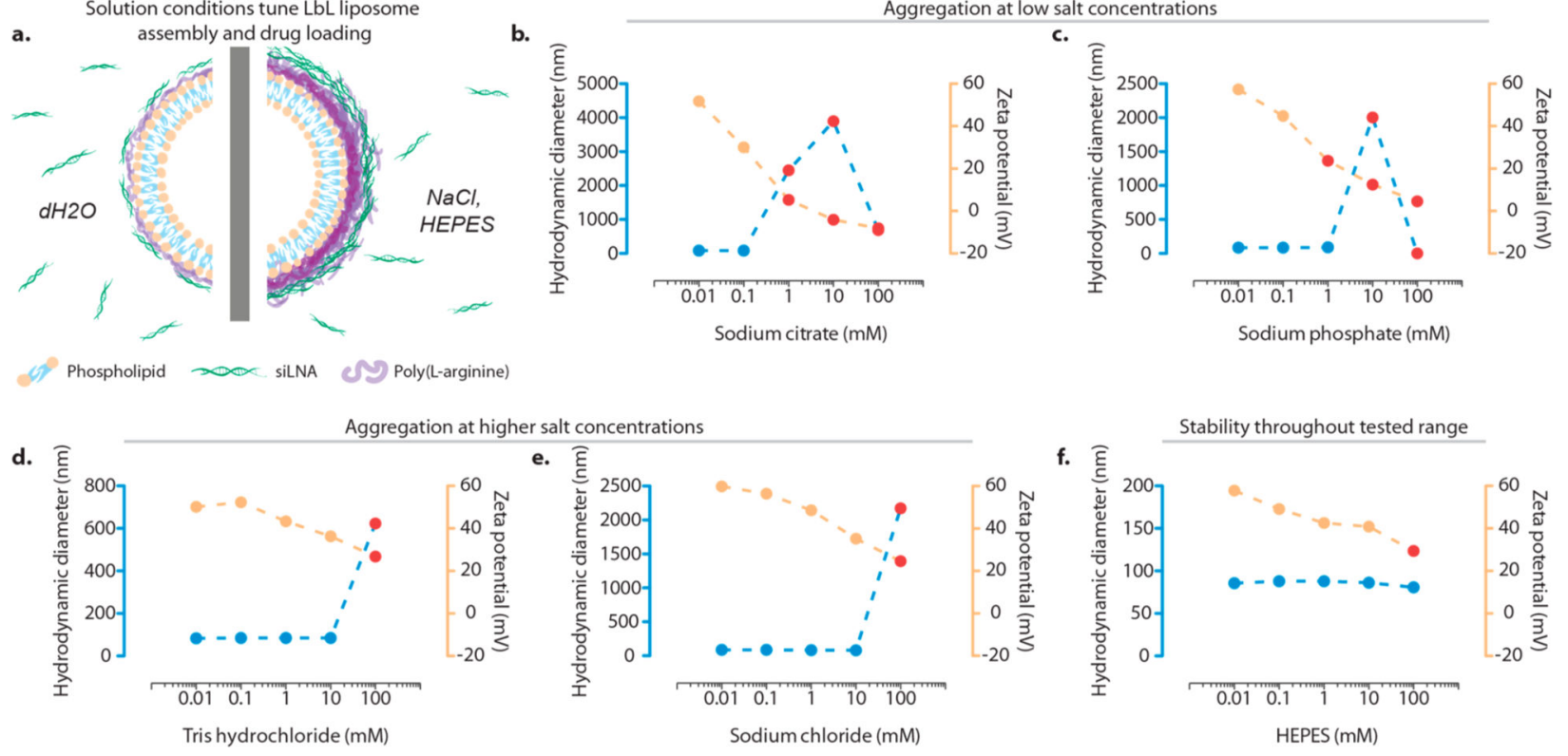

\section{Figure 2.}

Layer-by-layer assembly onto liposomal substrates is affected by both salt concentration and salt identity, with notable differences in colloidal stability noted based on salt valency and size. (a) Schematic of the LbL process of layering the polycation onto liposomes in the absence of salt (using deionized water, DI $\mathrm{H}_{2} \mathrm{O}$ ) and in the presence of salts compatible with the $\mathrm{LbL}$ process ( $\mathrm{NaCl}$ and HEPES). Multivalent ions like (b) sodium citrate and (c) sodium phosphate destabilize PLR adsorption. Monovalent species (d) Tris and (e) $\mathrm{NaCl}$ are less destabilizing but cause flocculation at moderate concentrations (100 mM). Zwitterionic (f) HEPES was tolerated at all concentrations used, with only a slight dip in the zeta potential at $100 \mathrm{mM}$. Particle instability is denoted using red data points. Size and polydispersity data were acquired by dynamic light scattering (DLS), and zeta potential data were measured using laser Doppler electrophoresis. 
Tetralayer LbL liposome siLNA encapsulation

a. Purity $\rightarrow C$ W $C$ W $C$ C W $C$ W

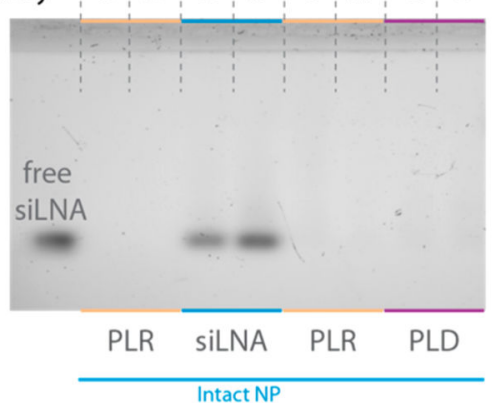

PLR layered on top of siLNA prevents release due to electrophoresis

b.

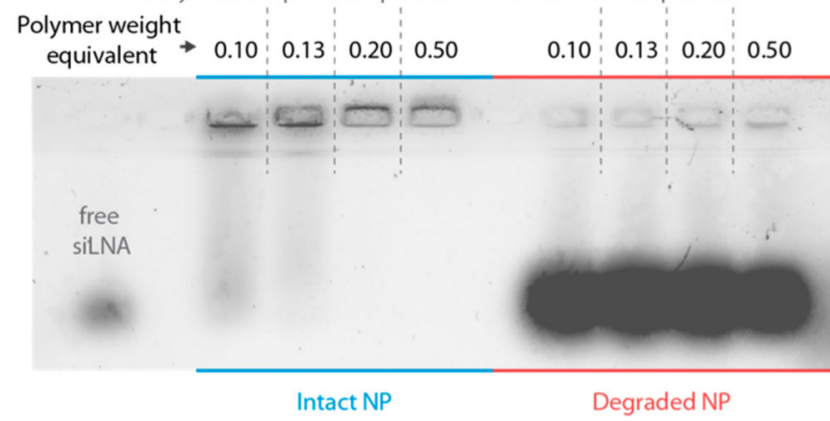

Figure 3.

LbL assembly encapsulates siLNA into NPs, and additional layers improve the stability of the nanocomposite. (a) Agarose gel analysis of each layer of the LbL NP synthesis carried out in $\mathrm{pH} 7.5$ titrated water. siLNA migrates into the gel when left as the terminal layer, but migration into gel is not seen after capping with additional layers. (b) Agarose gel analysis of liposome/PLR/siLNA/PLR LbL NPs formed at varying PLR weight equivalents. Both intact (blue) and degraded (red) NPs are shown. 
a.
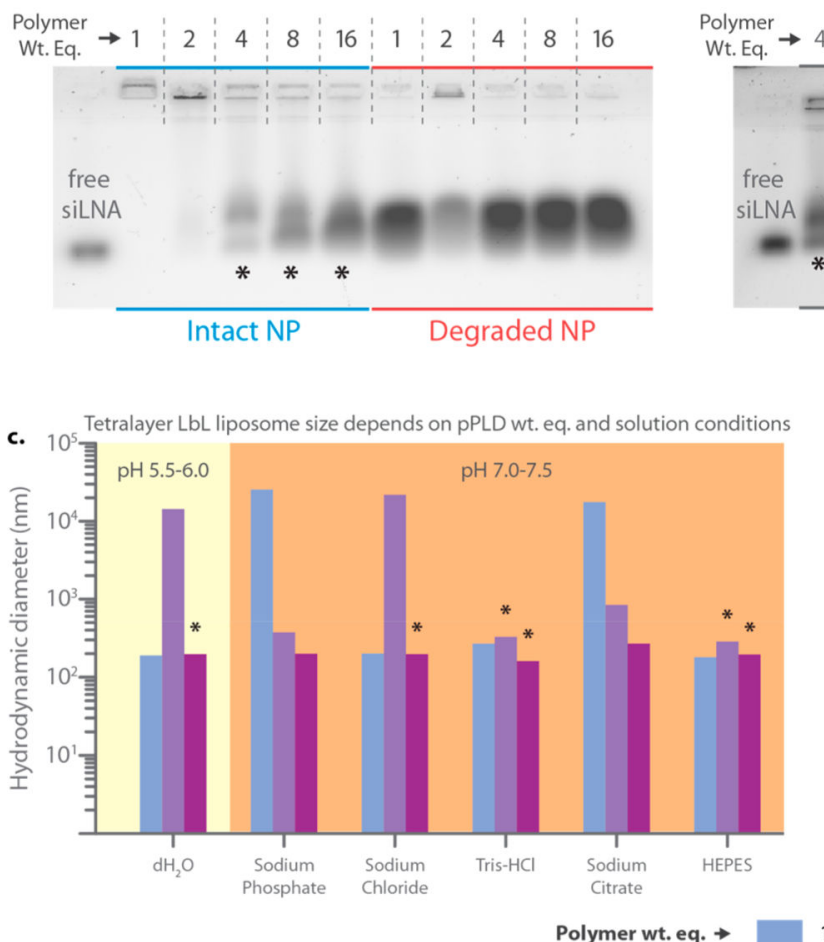

b.

Polymer

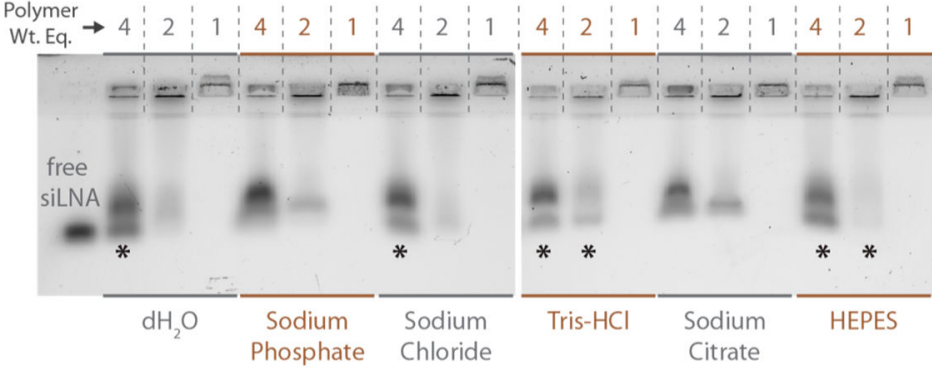

d. 60 Charge conversion depends on pPLD wt. eq. and solution conditions

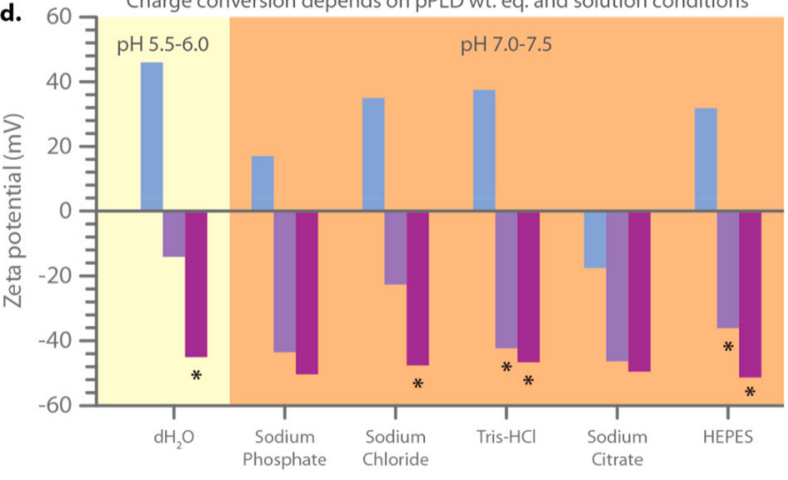

Figure 4.

Solution conditions tune layering efficiency and allow incorporation of pPLD without disrupting underlying siLNA layers. (a) Agarose gel analysis of pPLD titrations onto siLNAcontaining LbL NPs in DI $\mathrm{H}_{2} \mathrm{O}$. Increasing pPLD wt. eq. corresponds to siLNA displacement. Asterisks denote stable formulations with respect to particle size and zeta potential. (b) Agarose gel analysis of pPLD titrations onto liposome/PLR/siLNA/PLR NPs in DI $\mathrm{H}_{2} \mathrm{O}$ and salt solutions. Asterisks denote stable formulations. Tris and HEPES permit successful LbL assembly at lower pPLD wt. eq. (c) Size data of the nanoparticles formulated during the titration of the final pPLD layer. Asterisks denote stable formulations. (d) Zeta potential measurements for the same titration series. Asterisks denote stable formulations. Size and polydispersity data were acquired by DLS, and zeta potential data were measured using laser Doppler electrophoresis. 

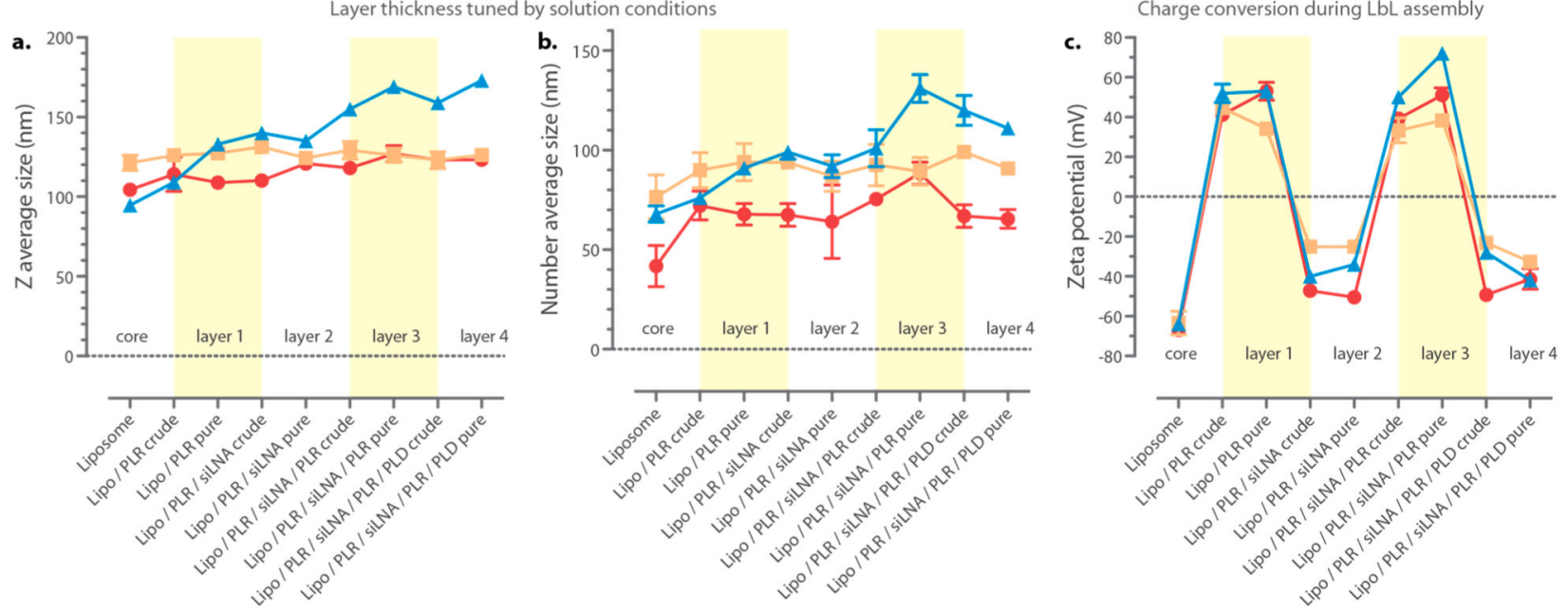

$\mathrm{dH} 2 \mathrm{O}-$ Titrated water $(\mathrm{pH} 7.5) \longrightarrow$ HEPES and $\mathrm{NaCl}$

Layering efficiency with differing solution conditions

d.

\begin{tabular}{|c|c|c|c|c|}
\cline { 2 - 5 } \multicolumn{1}{c|}{} & $\begin{array}{c}\text { layer } \\
1\end{array}$ & $\begin{array}{c}\text { layer } \\
2\end{array}$ & $\begin{array}{c}\text { layer } \\
3\end{array}$ & $\begin{array}{c}\text { layer } \\
4\end{array}$ \\
\hline Polymer & PLR & siLNA & PLR & PLD \\
\hline $\mathrm{dH}_{2} \mathrm{O}$ wt. eq. & 0.20 & 2.00 & 1.50 & 1.00 \\
\hline Titrated water wt. eq. & 0.05 & 0.07 & 0.10 & 0.03 \\
\hline HEPES and $\mathrm{NaCl}$ wt. eq. & 0.50 & 1.00 & 0.50 & 0.40 \\
\hline
\end{tabular}
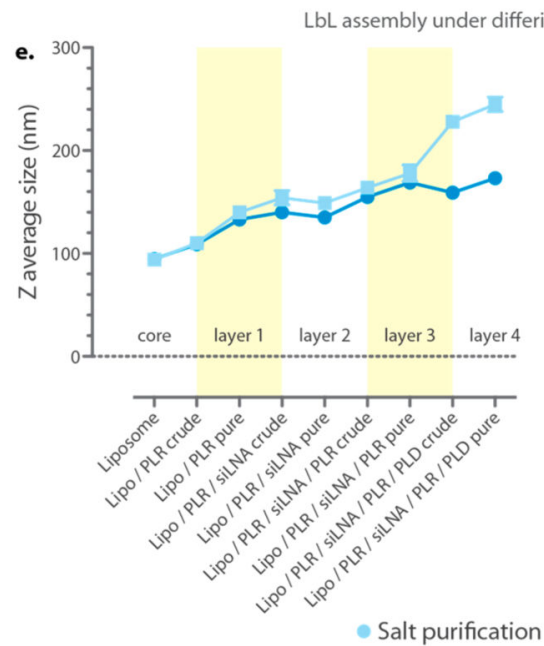
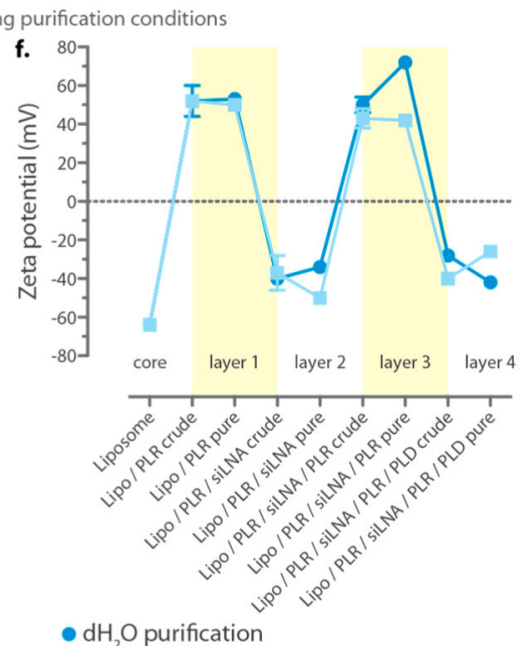

Figure 5.

Solution conditions during assembly and purification influence layering efficiency and LbL liposome size. The (a) $z$-average diameter, (b) number-average diameter, and (c) zeta potential of LbL liposomes prepared in deionized water $\left(\mathrm{DI} \mathrm{H}_{2} \mathrm{O}\right.$ ), $\mathrm{pH} 7.5$ titrated water (20 $\mathrm{mM} \mathrm{Na}_{2} \mathrm{HPO}_{4}$ ), or $25 \mathrm{mM}$ HEPES $20 \mathrm{mM} \mathrm{NaCl}$. The HEPES/ $\mathrm{NaCl}$ condition is buffer exchanged to water during the interlayer purification steps. (d) Weight equivalents of polyelectrolyte required to achieve stable charge conversion at each layer. The (e) $z$-average diameter and (f) zeta potential of LbL liposomes prepared in $25 \mathrm{mM}$ HEPES $20 \mathrm{mM} \mathrm{NaCl}$ purified in either $25 \mathrm{mM}$ HEPES $20 \mathrm{mM} \mathrm{NaCl}$ or DI $\mathrm{H}_{2} \mathrm{O}$. Size and polydispersity data were acquired by DLS, and zeta potential data were measured using laser Doppler electrophoresis. Error bars represent the standard deviation of three technical replicates. 
a.

Solution conditions

\begin{tabular}{|c|c|c|}
\hline Condition & Layer deposition & Interlayer purification \\
\hline 1 & $\mathrm{pH} 7.5$ titrated $\mathrm{H}_{2} \mathrm{O}\left(20 \mu \mathrm{M} \mathrm{Na}_{2} \mathrm{HPO}_{4}\right)$ & $\mathrm{pH} 7.5$ titrated $\mathrm{H}_{2} \mathrm{O}\left(20 \mu \mathrm{M} \mathrm{Na}_{2} \mathrm{HPO}_{4}\right)$ \\
\hline 2 & 25 mM HEPES & 25 mM HEPES \\
\hline 3 & $25 \mathrm{mM}$ HEPES $20 \mathrm{mM} \mathrm{NaCl}$ & $25 \mathrm{mM}$ HEPES $20 \mathrm{mM} \mathrm{NaCl}$ \\
\hline 4 & $25 \mathrm{mM}$ HEPES $20 \mathrm{mM} \mathrm{NaCl}$ & $\mathrm{dH}_{2} \mathrm{O}$ \\
\hline
\end{tabular}

b.

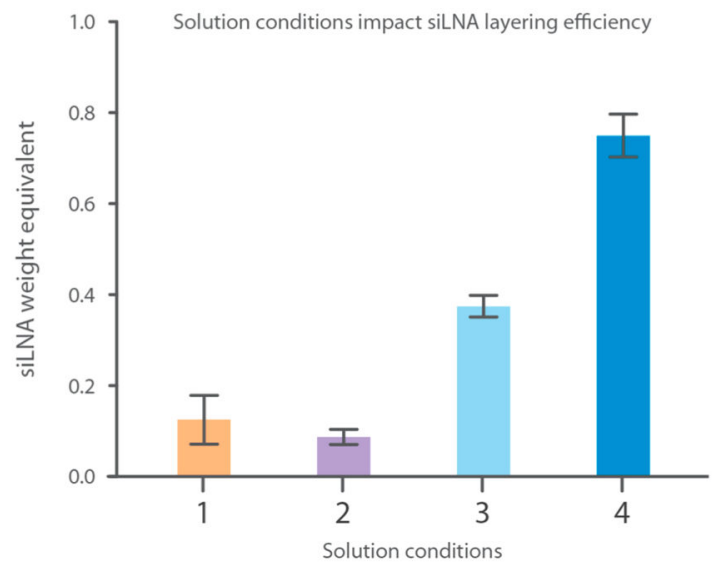

c.

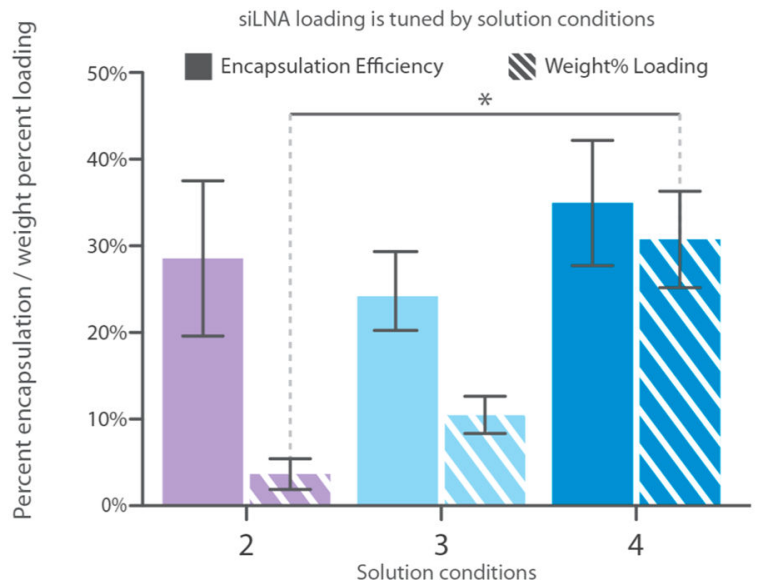

Figure 6.

Solution conditions during both assembly and purification tune siLNA loading of the LbL film. (a) The synthetic solution conditions tested in these experiments are defined. (b) LbL NPs were prepared under the defined solution conditions, and changes in the optimal weight equivalent of polyelectrolyte needed to achieve a stable colloid were recorded and averaged in addition to the amount of siLNA added per milligram of lipid (LbL NP core). (c) The encapsulation effciency of siLNA in LbL NPs synthesized in different solution conditions was assessed. Data are representative of at least three independent syntheses for each condition. Error bars represent SEM. All statistical tests were performed using one-way ANOVA (alpha $=0.05)$, with the Tukey post-test, on PRISM graphing software. 


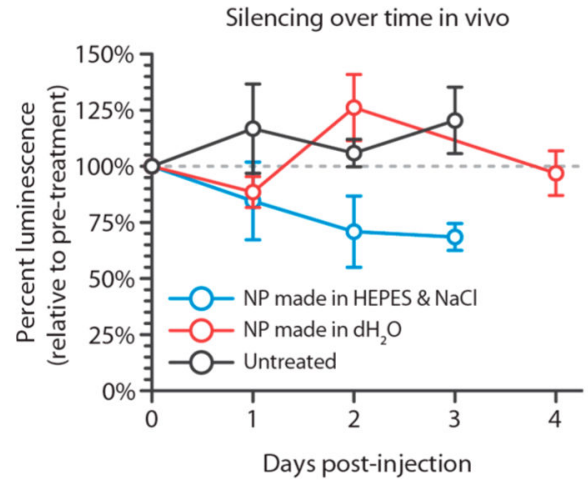

b.

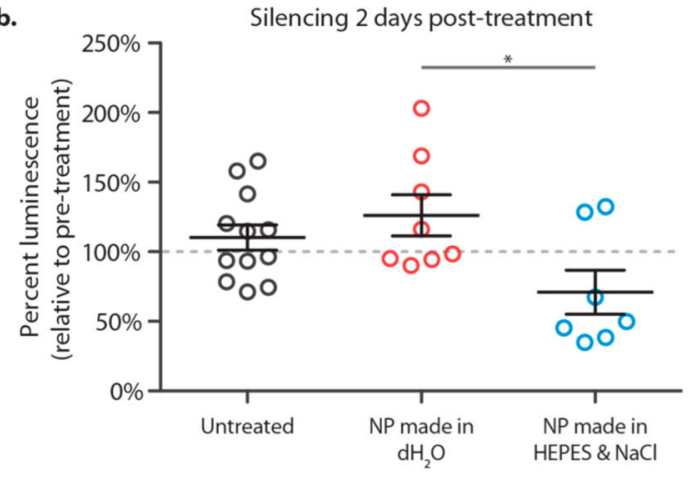

c.

Pre-treatment

2 days post-treatment

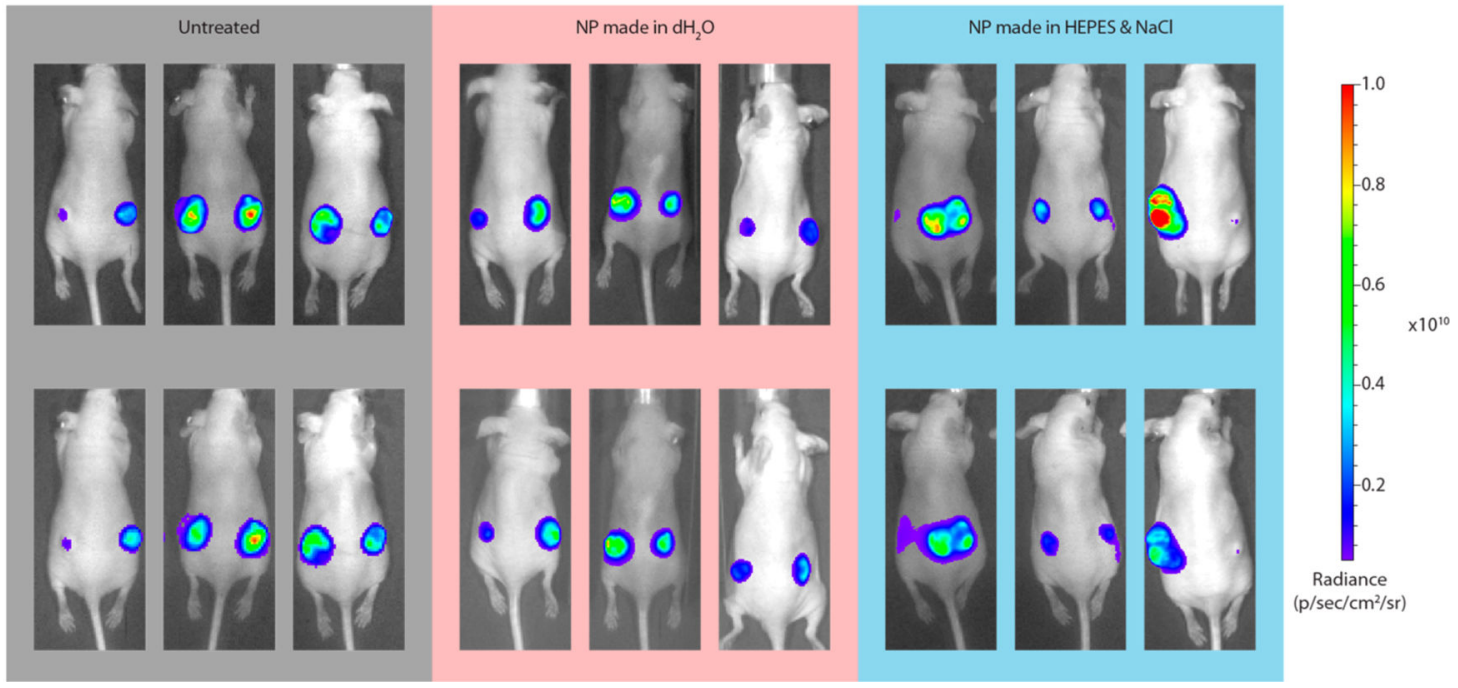

Figure 7.

Optimized solution conditions produce LbL siLNA carriers that mediate improved transfection of model genes in ovarian cancer in vivo. (a) NCR nude mice bearing luciferized subcutaneous flank tumor xenografts were intravenously injected with nanoparticles to provide a $0.5 \mathrm{mg} / \mathrm{kg}$ dose of siLNA. Luciferase signal was determined using IVIS whole-animal imaging. (b) Luciferase expression data from 2 days post-treatment. (c) Representative whole-animal luminescence images of mice treated with LbL liposomes prior to treatment and 2 days post-treatment. Error bars represent SD of triplicates in panel a and SEM of at least $n=6$ in panels $\mathrm{b}$ and $\mathrm{c}$. All statistical tests were performed using one-way ANOVA (alpha $=0.05$ ), with the Tukey post-test, on PRISM graphing software. 